\title{
Immunological mechanisms in the reaction between drugs and the skin
}

\author{
J. L. TURK \\ Department of Pathology, Royal College of Surgeons of England
}

The adverse effects of a wide range of chemical therapeutic agents are manifested in the skin. In fact, the term 'drug reaction' is almost synonymous in the minds of most physicians with the development of an 'allergic rash'. However, most chemical substances that elicit such a reaction are of too low molecular weight to be able to induce an immune response in isolation and have been considered, since the time of Ehrlich, to act as immunogens by combination in vivo with higher molecular weight protein molecules. Landsteiner and Jacobs (1935) showed that they could immunize animals with chloro- and nitrosubstitute benzenes only if these were attached covalently to proteins.

Certain sensitizers such as picric acid, penicillin, azo dyes and sulphonamides are, however, incapable of conjugating with protein. It was therefore suggested that products of metabolism, formed in the body, might link covalently with protein (Landsteiner and di Somma, 1940; Mayer, 1954). For instance, Landsteiner and di Somma (1940) postulated that picric acid was reduced in vivo to picramic acid which could bind covalently to protein but Chase and Maguire (1974) have found picramic acid to be a poor sensitizer. As picric acid can bind reversibly to albumin by hydrogen bonding, it became questionable whether a covalent linkage was essential for sensitization by this agent. Sensitization with picric acid shows a number of characteristics which distinguishes it from sensitization with other agents which are linked covalently. For instance, cross reactions between picric acid and picryl chloride are unusual. Thus, animals sensitized with picric acid are sensitive to picryl chloride by contact but not to the intradermal injection of the free chemical or picrylated protein. Moreover, delayed hypersensitivity to picric acid in intradermal tests may be transferred by cells without the recipients developing positive contact reactions to picric acid. Thus it would appear possible that compounds of this nature may form immunogens with weaker links to the carrier, such as hydrogen bonding or Van der Waal's forces. Such carriers could be proteins or polysaccharides.
Azo dyes and sulphonamides are thought to be metabolized in vivo to compounds with a quinone structure and the penicilloyl group is recognized as being the main antigenic determinant involved in hypersensitivity to penicillin (Levine and Ovary, 1961). However, with these last drugs it is still likely that the immunogen is formed as a result of a covalent link with the carrier molecule.

Drugs acting as immunogens can cause manifestations either as a result of local allergic tissue damage or as the result of a more generalized effect. The basic immunological mechanisms behind such reactions are the same and depend on whether the response is mediated by the products of $T$ or $B$ lymphocytes. Whether T or B cells preferentially respond may be due to whether the antigen is fixed or whether it is present in solution. A chemical com? pound bound to the skin tends to provoke a pre- $\rightleftharpoons$ dominantly $\mathrm{T}$-cell response as in the reaction to skin allografts. These are due to cell-mediated reactions and the direct interaction between specifically sensitized lymphocytes and the target antigen. An antigen present in solution in the extracellular fluid tends to stimulate predominantly $\mathbf{B}$ cells and the reaction is mediated through the interaction of antibodies with the target antigen. These are of two types. One type is predominantly urticarial and is produced by IgEtype antibody activating mast cells and leading to the release of vasoactive amines and other pharmacological agents from these cells. Such reactions are occasionally associated with the systemic manifestations of a generalized anaphylaxis. The other type is due to immune complexes and involves IgG or $\operatorname{IgM}$ antibody and the activation of the complement cascade. The effect of complement on the skin is due to an effect on the blood vessels causing an 'allergic vasculitis' with marked polymorphonuclear leucocyte infiltration. Local immune complex formation in the skin leads to a reaction that is described as the Arthus phenomenon with erythema, oedema and haemorrhage resulting occasionally in local skin necrosis (necrotizing vasculitis). If it is due to the deposition of circulating complexes, there may also 
be an associated glomerulonephritis (HenochSchonlein purpura) or arthritis. In this paper I shall concentrate on some aspects of drug interaction in delayed hypersensitivity.

\section{T Lymphocyte-mediated Hypersensitivity Reactions}

Drugs may elicit cell-mediated immune reactions by direct contact or by the production of a generalized exanthem. Contact sensitivity can be shown to be due to antibiotics such as penicillin, streptomycin and neomycin. Similar delayed hypersensitivity reactions can also be demonstrated with drugs such as neoarsphenamine injected intradermally.

Much of our knowledge of this type of mechanism has been derived from experimental models using contact sensitivity in the guinea pig to 2, 4-dinitrofluorobenzene (DNFB) or delayed hypersensitivity to the intradermal injection of ovalbumin. With both these systems, it has been possible to identify a population of 'suppressor' as well as 'effector' cells and to demonstrate that the final effect is the result of a balance between these two (fig 1). Both effector $T$ cells and suppressor cells, which may be $B$ cells or $T$ cells, have similar specificities. It is likely that only effector cells secrete the non-specific pharmacological agents that have been given the generic name of 'lymphokines'. The main action of lymphokines is to activate macrophage enzyme systems and it is probably the leakage of these enzymes from the cells

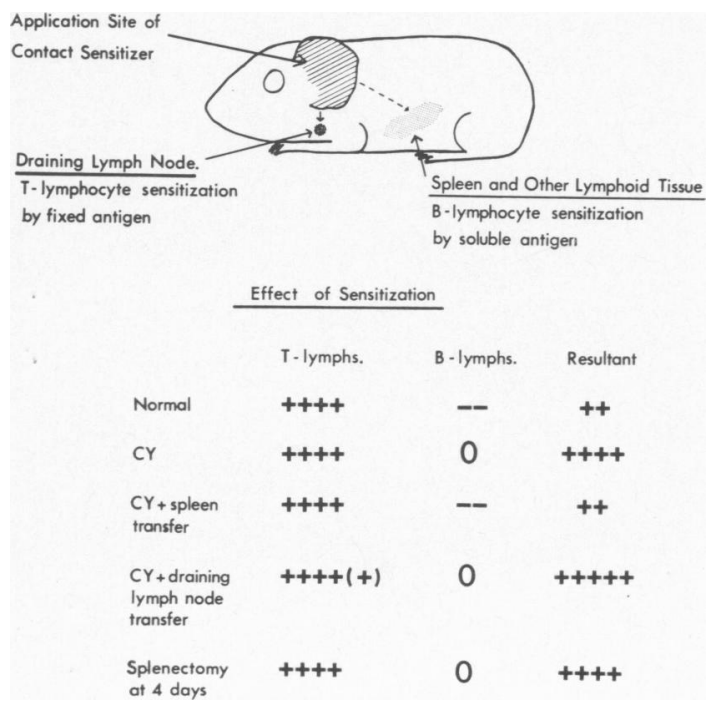

Fig 1 Proposed result of the interaction between $B$ lymphocytes and T lymphocytes in contact sensitivity in the guinea pig.

$+=$ effector cell action, $-=$ suppressor cell action. that causes the local tissue damage that results in the inflammatory process. These agents, which are proteins of molecular weight in the region of 40000 , are chemotactic for macrophages and produce changes in the electrostatic charge on the surface of these cells and thus reduce the ability of macrophages to migrate. It could be that the main difference between suppressor and effector cells is that the former are unable to secrete lymphokines and activate macrophages and as a result compete with effector cells for the antigen at the site of reaction. Reactions of this type have been shown to be characterized specially by an infiltration with basophilic leucocytes. This might indicate that under certain circumstances, in the guinea pig, suppressor cells act by local secretion of a $\gamma_{1}$ or $\gamma_{E}$ homocytotropic antibody.

Suppressor cells are temporarily eliminated by treating the guinea pigs with cyclophosphamide in a single dose of $300 \mathrm{mg} / \mathrm{kg}$ injected three days before sensitization (Turk, Parker, and Poulter, 1972). This, incidentally, will also reduce the level of circulating basophils. Under these conditions, effector cells will act in an unrestrained manner and produce reactions that are up to twice as intense and last twice as long as those in normally sensitized animals. This type of reactivity can be returned to normal by injection of cell suspensions containing adequate numbers of suppressor cells. These cells may be found in spleen and peritoneal exudate suspensions from normal animals sensitized seven days previously. Furthermore, in the guinea pig it can be shown, using specific anti-immunoglobulin-coated columns, that the active cells in these suspensions belong to a B rather than a $T$ cell population (Katz, Parker, and Turk, 1974).

It is likely, therefore, that in every immunological situation the resultant effect depends on the relative balance between effector and suppressor cells. Both types of cell appear to be produced, however mild the stimulus. In some situations effector cell production may be so great that elimination of suppressor cells will have no effect on the result. However, under other conditions suppressor cell populations will form such a high proportion of the cells that they inhibit further effector cell production and produce a state of permanent immunological unreactiveness. This is a situation that was frequently referred to in the past as immunological unresponsiveness or tolerance.

Such a state of unresponsiveness to 2, 4-dinitrochlorobenzene (DNCB) can be produced in the guinea pig by the intravenous injection of $300 \mathrm{mg} / \mathrm{kg}$ dinitrobenzene sulphonic acid sodium salt $\left(\mathrm{DNBSO}_{3}\right.$ $\mathrm{Na}$ ), which is a water-soluble dinitrophenylating compound, twice at 14-day intervals before sensitizing with DNCB. Similar unresponsiveness can be induced by feeding DNCB to animals over a period 
of months. In both these situations one can also demonstrate a failure of specific $\mathrm{T}$ cell proliferation in the draining lymph nodes four days after attempted sensitization. It can be shown by the suitable use of the drug cyclophosphamide that the unresponsiveness in these two models is a process of active suppression. If cyclophosphamide $(300 \mathrm{mg} / \mathrm{kg})$ is given three days before attempted sensitization, unresponsiveness can be reversed (Polak and Turk, 1974). However, if one waits 14 days after cyclophosphamide before attempting sensitization, animals become unresponsive again. This indicates that cyclophosphamide is temporarily inhibiting the action of the suppressor cells. As the effect of cyclophosphamide is specific to those lymphocytes which are in a process of DNA synthesis, it could be that it works on the population of cells which are turning over more rapidly as a result of antigenic stimulation. These may be T or B cells and B cells are normally susceptible to the effect of cyclophosphamide even in the resting state. This would indicate that in this system immunological 'tolerance' or unresponsiveness is an active state due to antigenic stimulation and there would appear to be no evidence for the hypothesis that the tolerant state is produced by elimination of a specific clone of responsive cells by the high dose of antigen injected.

Another method for producing a state of hyporeactivity to DNFB is by prior painting of the skin with the cross-reacting chemical agent 2, 4-dinitrothiocyanate benzene(DNTB). 2, 4-Dinitrothiocyanate benzene fails to sensitize animals when painted on the skin but after such treatment they become specifically hyporeactive to DNFB. Such hyporeactivity is not reflected by a failure of $T$ cell proliferation in the draining lymph node and treatment of the animals with cyclophosphamide will temporarily reverse this hyporeactive state. This indicates that there is involvement of suppressor cells acting on the efferent side of the immunization arc. In further experiments, it has been possible to demonstrate suppressor cell activity in the spleen and in induced peritoneal exudates by showing that transfusion of these cells to other sensitized animals would reduce their reactivity. In these experiments we used as recipients animals whose reactivity had been increased by pretreatment with cyclophosphamide three days before sensitization with DNFB (Sommer, Parker, and Turk, 1975).

Thus two types of unresponsiveness can be demonstrated. In one, there is central suppression of $T$ cell responsiveness, and this can be produced by intravenous injection of large doses of sensitizer or feeding this by mouth. In the second, there is peripheral suppression of activity and this be can induced by painting the skin with a cross-reacting agent such as DNTB.

\section{Fixed Drug Eruptions}

One of the more common manifestations of drug allergy in the skin is the so-called 'fixed drug eruption'. This localized eruption always occurs at the same time and the same site after systemic absorption of the sensitizing agent. Among the drugs that are well known to produce this type of reaction are barbiturates, sulphonamides, phenolphthalein and phenazone. There is little concrete evidence available as to why the eruption always occurs at one particular site. However, during experiments on contact sensitization with potassium dichromatc $\left(\mathrm{K}_{2} \mathrm{Cr}_{2} \mathrm{O}_{7}\right)$ it was found that if an animal was given the chemical intravenously, the site of the reaction would be that of previous induced contact (Polak and Turk, 1968). Flare-up reactions can even be induced in skin test sites that have lain dormant for three months. A flare up can occur with a dose as low as $0.2 \mathrm{mg} / \mathrm{kg}$ body weight absorbed systemically. Histological examination of these reactions indicated that they have a polymorphonuclear rather than a mono-

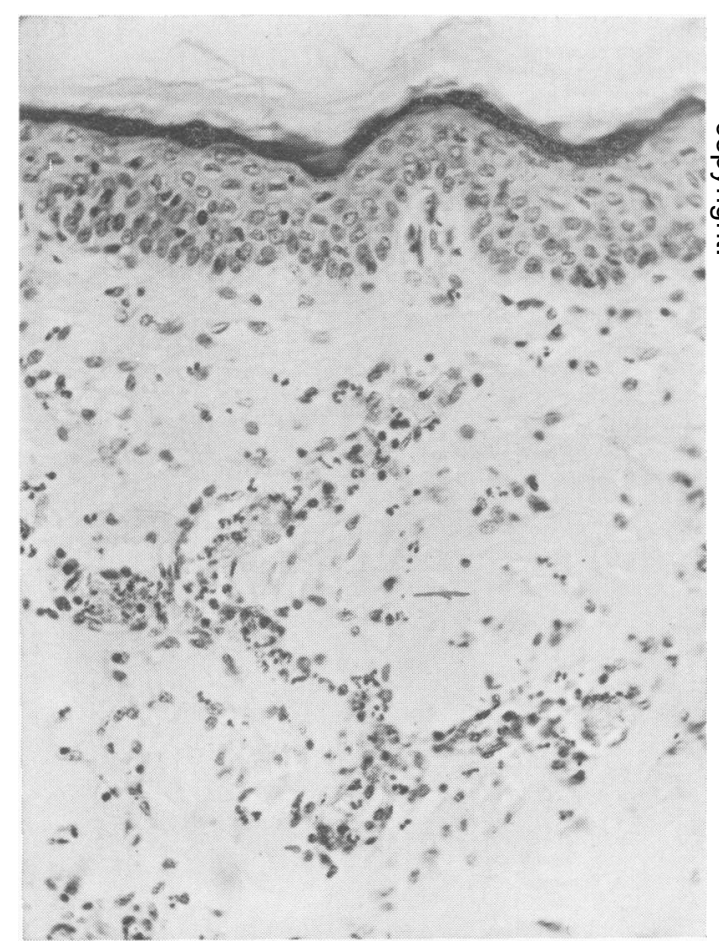

Fig 2 Section of guinea pig skin which had been the site of a contact sensitivity reaction to $0.5 \%$ $\mathrm{K}_{2} \mathrm{Cr}_{2} \mathrm{O}_{7}$ two weeks previously. Flare-up reaction six hours after intravenous injection of $20 \mathrm{mg} / \mathrm{kg} \mathrm{K}_{2} \mathrm{Cr}_{2} \mathrm{O}_{7} . \mathrm{H} \& \mathrm{E}$ $\times 140$ (Polak and Turk, 1968). 
nuclear cell infiltrate (fig 2), and the time course was more like that of an Arthus reaction, reaching maximum intensity between four and six hours. Moreover, animals made specifically unresponsive for contact sensitivity would still show these 'flare-up' reactions at sites of previous contact reactions. All these points indicate that this phenomenon is B cell rather than $T$ cell mediated. It could be that $B$ cells come into the test site as part of the non-specific inflammatory infiltrate, and are there sensitized with antigen, and remain at the site until antigen reaches them at a later date and triggers the local eruption. These B cells may be in the form of plasma cells secreting antibody locally or could be $B$ cells with specific antibody on their surface. Such flare-up reactions can also occur at the site of a subliminal contact even though there is no macroscopic reaction.

\section{Summary}

Many reactions in the skin caused by drugs would appear to be due to immunological mechanisms. Simple chemical sensitizers become antigenic after combination with carrier proteins. The bonds between the hapten and the protein are not necessarily covalent, and it is likely that weaker linkages such as hydrogen bonding or Van der Waal's forces may be involved. Reactions may be due to humoral antibody or cell-mediated immunity. Experimental models are discussed in which there is competition between B suppressor cells and $\mathrm{T}$ effector cells. In these situations, drugs that affect the suppressor cell system specifically can reverse a state of immunological tolerance or increase the intensity of a chemical sensitivity. Finally, the mechanism of fixed drug eruptions is discussed. It is suggested that the re- actions which follow the systemic absorption of the chemical and always occur at this same site are due to B cells or B cell products that remain from a previous delayed hypersensitivity reaction to the same antigen. These cells would have arrived initially as part of the non-specific inflammatory infiltrate. The initial reaction may even have been subliminal in intensity.

\section{References}

Chase, M. W., and Maguire, H. C., Jr. (1974). Further studies on sensi • tization to picric acid. In Contact Hypersensitivity in Experimental Animals, Monographs in Allergy, Vol. 8, edited by D. Parker and J. L. Turk, pp. 1-12. Karger, Basle.

Katz, S. I. Parker, D., and Turk, J. L. (1974). B cell suppression of delayed hypersensitivity reactions. Nature (Lond.), 251, 550-551

Landsteiner, K., and Di Somma, A. A. (1940). Studies on the sensitization of animals with simple chemical compounds. VIII. Sensitization to picric acid: subsidiary agents and mode of sensitization. J. exp. Med., 72, 361-366.

Landsteiner, K., and Jacobs, J. (1935). Studies on the sensitization of animals with simple chemical compounds. J. exp. Med., 61, 643-656.

Levine, B. B., and Ovary, Z. (1961). Studies on the mechanism of formation of the penicillin antigen. III The $\mathrm{N}$-(D-a-benzylpenicilloyl) group as an antigenic determinant responsible for hypersensitivity to Penicillen G. J. exp. Med., 114, 875-904.

Mayer, R. L. (1954). Group sensitization to compounds of quinone structure and its biochemical basis: role of these substances in cancer. Progr. Allergy, 4, 79-172.

Polák, L., and Turk, J. L. (1968). Studies on the effect of systemic administration of sensitizers in guinea pigs with contact sensitivity to inorganic metal compounds. II The flare-up of previous test sites of contact sensitivity and the development of a generalized rash. Clin. exp. Immunol., 3, 253-262.

Polák, L., and Turk, J. L. (1974). Reversal of immunological tolerance by cyclophosphamide through inhibition of suppressor cell activity. Nature (Lond.), 249, 654-656.

Sommer, G., Parker, D., and Turk, J. L. (1975). Epicutaneous induction of hyporeactivity in contact sensitization: demonstration of suppressor cells induced by contact with 2, 4-dinitrothiocyanate benzene. Immunology, 29, 517-525.

Turk, J. L., Parker, D., and Poulter, L. W. (1972). Functional aspects of the selective depletion of lymphoid tissue by cyclophosphamide. Immunology, 23, 493-501. 